\title{
Exact Coulomb cutoff technique for supercell calculations
}

\author{
Carlo A. Rozzi, ${ }^{1,2}$ Daniele Varsano, ${ }^{3,2}$ Andrea Marini, ${ }^{4,2}$ Eberhard K. U. Gross, ${ }^{1,2}$ and Angel Rubio ${ }^{1,3,2}$ \\ ${ }^{1}$ Institut für Theoretische Physik, Freie Universität Berlin, Arnimallee 14, D-14195 Berlin, Germany \\ ${ }^{2}$ European Theoretical Spectroscopy Facility (ETSF), D-14195 Berlin, Germany and E-20018 San Sebastián, Spain \\ ${ }^{3}$ Departamento de Física de Materiales, Facultad de Ciencias Químicas, UPV/EHU, Centro Mixto CSIC-UPV/EHU \\ and Donostia International Physics Center, E-20018 San Sebastián, Spain \\ ${ }^{4}$ Dipartimento di Fisica, Università "Tor Vergata”, Roma, Italy
}

(Received 23 December 2005; revised manuscript received 31 March 2006; published 26 May 2006)

\begin{abstract}
We present a reciprocal space analytical method to cut off the long range interactions in supercell calculations for systems that are infinite and periodic in one or two dimensions, generalizing previous work to treat finite systems. The proposed cutoffs are functions in Fourier space, that are used as a multiplicative factor to screen the bare Coulomb interaction. The functions are analytic everywhere except in a subdomain of the Fourier space that depends on the periodic dimensionality. We show that the divergences that lead to the nonanalytical behavior can be exactly canceled when both the ionic and the Hartree potential are properly screened. This technique is exact, fast, and very easy to implement in already existing supercell codes. To illustrate the performance of the scheme, we apply it to the case of the Coulomb interaction in systems with reduced periodicity (as one-dimensional chains and layers). For these test cases, we address the impact of the cutoff on different relevant quantities for ground and excited state properties, namely: the convergence of the ground state properties, the static polarizability of the system, the quasiparticle corrections in the GW scheme, and the binding energy of the excitonic states in the Bethe-Salpeter equation. The results are very promising and easy to implement in all available first-principles codes.
\end{abstract}

DOI: 10.1103/PhysRevB.73.205119

PACS number(s): 02.70.-c, 31.15.Ew, 71.15.Qe

\section{INTRODUCTION}

Plane-wave expansions and periodic boundary conditions have been proven to be a very effective way to exploit the translational symmetry of infinite crystal solids, in order to calculate the properties of the bulk, by performing the simulations in one of its primitive cells only. ${ }^{1}$ The use of plane waves is motivated by several facts. First, the translational symmetry of the potentials involved in the calculations is naturally and easily accounted for in reciprocal space, through the Fourier expansion. Second, very efficient and fast algorithms exist (such as ${ }^{2}$ FFTW) that allow us to calculate the Fourier transforms very efficiently. Third, the expansion in plane waves is exact, since they form a complete set, and it is only limited in practice by one parameter, namely, the maximum value of the momentum, that determines the size of the chosen set. In addition, the use of Bornvon Karmán periodic boundary conditions, independently of the adopted basis set, gives a conceptually easy (though artificial) way to eliminate the dependence of the properties of a specific sample on its surface and shape, allowing us to concentrate on the bulk properties of the system in the thermodynamic limit. ${ }^{3}$

However, mainly in the last decade, increasing interest has been developed in systems on the nanoscale, such as tubes, wires, quantumdots, biomolecules, etc., whose physical dimensionality is, for all practical purposes, less than three. ${ }^{4}$ These systems are still three-dimensional (3D), but their quantum properties are typical of a system confined in one or more directions, and periodic in the remaining ones. Other classes of systems with the same kind of reduced periodicity are the classes of the polymers, and of solids with defects.
Throughout this paper, we call $n \mathrm{D}$-periodic a $3 \mathrm{D}$ object that can be considered infinite and periodic in $n$ dimensions, and finite in the remaining 3- $n$ dimensions. In order to simulate this kind of system, a commonly adopted approach is the supercell approximation. ${ }^{1}$

In the supercell approximation, the physical system is treated as a fully 3D-periodic one, but a new unit cell (the supercell) is built in such a way that some extra empty space separates the periodic replicas along the direction(s) in which the system is to be considered as finite. This method makes it possible to retain all the advantages of plane-wave expansions and of periodic boundary conditions. Yet the use of a supercell to simulate objects that are not infinite and periodic in all the directions, leads to artifacts, even if much empty space is interposed between the replicas of the system in the nonperiodic dimensions.

In fact, the straightforward application of the supercell method always generates fictitious images of the original system, that can mutually interact in several ways, affecting the results of the simulation. It is well known that the response function of an overall neutral solid of molecules is not equal, in general, to the response of the isolated molecule and converges very slowly when the empty space in the supercell is progressively increased. ${ }^{7,8}$

For instance, the presence of higher order multipoles can make undesired images interact via the long range part of the Coulomb potential. In the dynamic regime, multipoles are always generated by the oscillations of the charge density. This is the case, for example, when we investigate the response of a system in the presence of an external oscillating electric field.

Things worsen when the unit cell carries a net charge, since the total charge of the infinite system represented by the supercell is actually infinite, while the charges at the 
surfaces of a finite, though very large system always generate a finite polarization field. This situation is usually normalized in the calculation by the introduction of a suitable compensating positive background charge. ${ }^{9}$

Another common situation in which the electrostatics is known to modify the ground state properties of the system occurs when a layered system is studied, and an infinite array of planes is considered instead of a single slab. Such an infinite array is in fact equivalent to an effective chain of capacitors..$^{10}$

These problems become particularly evident in all approaches that require the calculation of nonlocal operators or response functions, because, in these cases, a supercell, and its periodic images may effectively interact even if their charge densities do not overlap at all. This is the case, for example, in many-body perturbation theory calculations (MBPT), particularly in the self-energy calculations at the GW level. ${ }^{6,7}$

However we are generally still interested in the dispersion relations of the elementary excitations of the system along its periodic directions, and those are ideally treated using a plane-wave approach. Therefore, the best way to retain the advantages of the supercell formulation in plane waves, and to gain a description of systems with reduced periodicity free of spurious effects is to develop a technique to cut the Coulomb interaction off outside the desired region. This problem has been known for quite a long time and has appeared in different fields (condensed matter, classical physics, astrophysics, ${ }^{11}$ biology, ${ }^{12}$ particle physics $\left.{ }^{13}\right)$. Several different approaches have been proposed in the past to solve it. The aim of the present work is to focus on the widely used supercell schemes to show how the image interaction influences both the electronic ground state properties and the dynamical screening in the excited state of 0D-, 1D-, 2Dperiodic systems, and to propose an exact method to avoid the undesired interaction of the replicas in the nonperiodic directions.

The paper is organized as follows: in Sec. II, the basics of the plane wave method for solids are reviewed; in Sec. III, the method is outlined; in Sec. IV the treatment of the singularities is explained; in Sec. V, some applications of the proposed technique are discussed.

\section{THE 3D-PERIODIC CASE}

The main problem of electrostatics which we are confronted with here can be reduced to that of finding the electrostatic potential $V(\mathbf{r})$ that solves the Poisson equation for a given charge distribution $n(\mathbf{r})$, and given boundary conditions

$$
\nabla^{2} V(\mathbf{r})=-4 \pi n(\mathbf{r})
$$

In a finite system, the potential is usually required to be zero at infinity. In a periodic system, this condition is meaningless, since the system itself extends to infinity. Nevertheless, the general solution of Eq. (1) in both cases is known in the form of the convolution

$$
V(\mathbf{r})=\iiint_{\text {space }} \frac{n\left(\mathbf{r}^{\prime}\right)}{\left|\mathbf{r}-\mathbf{r}^{\prime}\right|} d^{3} \mathbf{r}^{\prime},
$$

which will be referred to from now on as the Hartree potential.

It might seem that the most direct way to build the solution potential for a given charge distribution is to compute the integral in real space. However problems immediately arise for infinite periodic systems. In fact, if we consider a periodic distribution of point charges located (for sake of simplicity) at the set of lattice points $\{\mathbf{L}\}$, then the periodic density can be reduced to an infinite sum over $\delta$ charge distributions $q \delta\left(\mathbf{r}-\mathbf{r}^{\prime}\right)$

$$
V(\mathbf{r})=\sum_{\{\mathbf{L}\}} \frac{q}{|\mathbf{r}-\mathbf{L}|},
$$

and the integral in Eq. (2) becomes an infinite sum as well. This sum is however in general only conditionally, and not absolutely convergent. ${ }^{14}$ The sum of Eq. (3) is a potential that is determined up to a constant for a neutral cell with zero dipole moment, while the corresponding sum for the electric field is absolutely convergent. A neutral cell with a nonzero dipole moment, on the other hand, gives a divergent potential, and an electric field that is determined up to an unknown constant electric field (the sum for the electric field is conditionally convergent in this case).

Even if, in principle, the surface terms should always be taken into account, in practice they are only relevant when we calculate energy differences between states with different total charge. These terms can be neglected in the case of a neutral cell whose lowest nonzero multipole is quadrupole. ${ }^{15}$ As in the present work we are interested in macroscopic properties of the periodic system, those surface effects are never considered in the discussion that follows. However these sample-shape effects play an important role for the analysis of different spectroscopies such as, for example, infrared and nuclear magnetic resonance.

A major source of computational problems is the fact that the sum in Eq. (3) is very slowly converging when it is summed in real space, and this fact has historically motivated the need for reciprocal space methods to calculate it. It was Ewald who first discovered that, by means of an integral transform, the sum can be split in two terms, and that if one is summed in real, while the other in reciprocal space, both of them are rapidly converging. ${ }^{16}$ The point of splitting is determined by an arbitrary parameter.

Let us now focus on methods of calculating the sum in Eq. (3) entirely in the reciprocal space.

Let us consider a 3D-periodic system with lattice vectors $\mathbf{L}$, and reciprocal lattice vectors $\mathbf{G}=\frac{2 \pi}{\mathbf{L}}$. The reciprocal space expression for the potential

$$
V(\mathbf{r})=\iiint_{\text {space }} n\left(\mathbf{r}^{\prime}\right) v\left(\left|\mathbf{r}-\mathbf{r}^{\prime}\right|\right) d^{3} \mathbf{r}^{\prime},
$$

can be written as 


$$
V(\mathbf{G})=n(\mathbf{G}) v(\mathbf{G}) .
$$

To transform the real space convolution of the density and the Coulomb potential into the product of their reciprocal space counterparts in Eq. (5), we have used the convolution theorem. Here $v(\mathbf{G})$ is the Fourier transform of the longrange interaction $v(\mathbf{r})$, evaluated at the point $\mathbf{G}$.

For the Coulomb potential, we have

$$
v(\mathbf{G})=\frac{4 \pi}{G^{2}} .
$$

Fourier transforming expression (5) back into real space we have, for a unit cell of volume $\Omega$

$$
V(\mathbf{r})=\frac{4 \pi}{\Omega} \sum_{\mathbf{G} \neq 0} \frac{n(\mathbf{G})}{G^{2}} \exp (i \mathbf{G} \cdot \mathbf{r})
$$

At the singular point $n_{x}=n_{y}=n_{z}=0$ the potential $V$ is undefined, but, since the value at $G=0$ corresponds to the average value of $V$ in real space, it can be chosen to be any number, corresponding to the arbitrariness in the choice of the static gauge (a constant) for the potential. Observe that the same expression can be adopted in the case of a charged unit cell, but this time, the arbitrary choice of $v(G)$ in $G=0$ corresponds to the use of a uniform background neutralizing charge.

\section{SYSTEMS WITH REDUCED PERIODICITY}

It has been shown ${ }^{17}$ that the slab capacitance effect mentioned in the Introduction is actually a problem that cannot be solved by just adding more vacuum to the supercell. This has initially led to the development of corrections to Ewald's original method, ${ }^{18}$ and subsequently to rigorous extensions in $2 \mathrm{D}$ and $1 \mathrm{D} .{ }^{19,20}$ The basic idea is to restrict the sum in reciprocal space to the reciprocal vectors that actually correspond to the periodic directions of the system. These approaches are in general of order $O\left(N^{2}\right),{ }^{21,22}$ where $N$ is the number of atoms, but they have been recently refined to order $O(N \ln N) .^{23,24}$ Another class of techniques, developed so far for finite systems, is based on the expansion of the interaction into a series of multipoles (fast multipole method). ${ }^{25-27}$ With this technique it is possible to evaluate effective boundary conditions for the Poisson's equations at the cell's boundary, so that the use of a supercell is not required at all, making it computationally very efficient for finite 25,26 and extended systems. ${ }^{27}$ Other known methods, typically used in molecular dynamics simulations, are the multipolecorrection method, ${ }^{28}$ and the particle-mesh method, ${ }^{29}$ whose review is beyond the scope of the present work. We refer the reader to the original works for details.

Unlike what happens for the Ewald sum, the method that we propose to evaluate the sum in Eq. (3) entirely relies on the Fourier space and amounts to screening the unit cell from the undesired effect of (some of) its periodic images. The basic expression is Eq. (5), whose accuracy is only limited by the maximum value $G_{\max }$ of the reciprocal space vectors in the sum. Since there is no splitting between real and reciprocal space, no convergence parameters are required.
Our goal is to transform the 3D-periodic Fourier representation of the Hartree potential of Eq. (5) into the modified one

$$
\tilde{V}(\mathbf{G})=\tilde{n}(\mathbf{G}) \widetilde{v}(\mathbf{G})
$$

such that all the interactions among the undesired periodic replicas of the system disappear. The present method is a generalization of the method proposed by Jarvis et al. ${ }^{30}$ for the case of a finite system.

In order to build this representation, we (1) define a screening region $\mathcal{D}$ around each charge in the system, outside of which there is no Coulomb interaction; (2) calculate the Fourier transform of the desired effective interaction $\widetilde{v}(r)$ that equals the Coulomb potential in $\mathcal{D}$, and is 0 outside $\mathcal{D}$

$$
\widetilde{v}(r)= \begin{cases}\frac{1}{r} & \text { if } r \in \mathcal{D} \\ 0 & \text { if } r \notin \mathcal{D} .\end{cases}
$$

Finally we must (3) modify the density $n(\mathbf{r})$ in such a way that the effective density is still 3D periodic, so that the convolution theorem can be still applied, but densities belonging to undesired images are not close enough to interact through $\tilde{v}(r)$.

The choice of the region $\mathcal{D}$ for step (1) is suggested by symmetry considerations, and it is a sphere (of radius $R$ ) for finite systems, an infinite cylinder (of radius $R$ ) for 1Dperiodic systems, and an infinite slab (of thickness $2 R$ ) for 2D-periodic systems.

Step (2) means that we have to calculate the modified Fourier integral

$$
\widetilde{v}(\mathbf{G})=\iiint_{\text {space }} \tilde{v}(r) e^{-i \mathbf{G} \cdot \mathbf{r}} d^{3} \mathbf{r}=\iiint_{\mathcal{D}} v(r) e^{-i \mathbf{G} \cdot \mathbf{r}} d^{3} \mathbf{r},
$$

since the modified $\tilde{v}$ potential is zero outside the domain $\mathcal{D}$. Still we have to avoid that two neighboring images interact by taking them far away enough from each other. Then step (3) means that we have to build a suitable supercell, and redefine the density in it.

Let us examine first step (2), i.e., the cutoff Coulomb interaction in reciprocal space. We know the expression of the potential when it is cutoff in a sphere. ${ }^{30}$ It is

$$
\widetilde{v}^{0 \mathrm{D}}(G)=\frac{4 \pi}{G^{2}}[1-\cos (G R)] .
$$

The limit $R \rightarrow \infty$ converges to the bare Coulomb term in the sense of a distribution, while, since $\lim _{G \rightarrow 0} \widetilde{v}^{0 \mathrm{D}}(G)=2 \pi R^{2}$, there is no particular difficulty in the origin. This scheme has been successfully used in many applications. ${ }^{14,26,30-32}$

The 1D-periodic case applies to systems with infinite extent in the $x$ direction, and finite in the $y$ and $z$ directions. The effective Coulomb interaction is then defined in real space to be 0 outside a cylinder with radius $R$ having its axis parallel to the $x$ direction. By performing the Fourier transformation we get the following expression for the cutoff Coulomb potential in cylindrical coordinates 


$$
\widetilde{v}^{1 \mathrm{D}}\left(G_{x}, G_{\perp}\right)=\int_{0}^{R} \int_{0}^{2 \pi} \int_{-\infty}^{+\infty} \frac{e^{-i\left(G_{x} x+G_{\perp} r \cos \theta\right)}}{\sqrt{r^{2}+x^{2}}} r d r d \theta d x,
$$

where $G_{\perp}=\sqrt{G_{y}^{2}+G_{z}^{2}}$. For $G_{x}, G_{\perp}>0$, the latter gives

$$
\begin{aligned}
\tilde{v}^{1 \mathrm{D}}\left(G_{x}, G_{\perp}\right) & =2 \int_{0}^{R} \int_{0}^{2 \pi} r K_{0}\left(G_{x} r\right) e^{-i G_{\perp} r \cos \theta} d r d \theta \\
& =4 \pi \int_{0}^{R} r K_{0}\left(G_{x} r\right) J_{0}\left(G_{\perp} r\right) d r
\end{aligned}
$$

and finally ${ }^{33}$

$$
\begin{aligned}
\tilde{v}^{1 \mathrm{D}}\left(G_{x}, G_{\perp}\right)= & \frac{4 \pi}{G^{2}}\left[1+G_{\perp} R J_{1}\left(G_{\perp} R\right) K_{0}\left(G_{x} R\right)\right. \\
& \left.-G_{x} R J_{0}\left(G_{\perp} R\right) K_{1}\left(G_{x} R\right)\right],
\end{aligned}
$$

where $J$ and $K$ are the ordinary and modified cylindrical Bessel functions.

It is clear that, since the $K$ functions damp the oscillations of the $J$ functions very quickly, for all practical purposes this cutoff function only acts on the smallest values of $G$, while the unscreened $\frac{4 \pi}{G^{2}}$ behavior is almost unchanged for the larger values.

Unfortunately, while the $J_{n}(\xi)$ functions have a constant value for $\xi=0$, and the whole cutoff is well defined for $G_{\perp}$ $=0$, the $K_{0}(\xi)$ function diverges logarithmically for $\xi \rightarrow 0$. Since, on the other hand, $K_{1}(\xi) \approx \xi^{-1}$ for small $\xi$

$$
\widetilde{v}^{1 \mathrm{D}}\left(G_{x}, G_{\perp}\right) \sim-\log \left(G_{x} R\right) \text { for } G_{\perp}>0, G_{x} \rightarrow 0^{+} .
$$

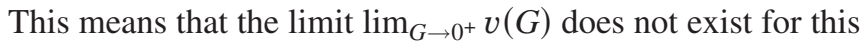
cutoff function, and the whole $G_{x}=0$ plane is ill defined. We will come back to the treatment of the singularities in the next section. We notice that this logarithmic divergence is the typical dependence one would get for the electrostatic potential of a uniformly charged 1D wire. ${ }^{34}$ It is expected that bringing charge neutrality in place would cancel this divergence (see below).

The 2D-periodic case, with finite extent in the $z$ direction, is calculated in a similar manner. The effective Coulomb interaction is defined in real space to be 0 outside a slab of thickness $2 R$ symmetric with respect to the $x y$ plane. In Cartesian coordinates, we get

$$
\widetilde{v}^{2 \mathrm{D}}\left(G_{x}, G_{y}, G_{z}\right)=\int_{-R}^{+R} \int_{-\infty}^{+\infty} \int_{-\infty}^{+\infty} \frac{e^{-i\left(G_{x} x+G_{y} y+G_{z} z\right)}}{\sqrt{x^{2}+y^{2}+z^{2}}} d z d y d x,
$$

and, calling $G_{\|}=\sqrt{G_{x}^{2}+G_{y}^{2}}$, for $G z, G_{\|}>0$, we get

$$
\begin{aligned}
\tilde{v}^{2 \mathrm{D}}\left(G_{\|}, G_{z}\right)= & 8 \int_{0}^{+R} \int_{0}^{+\infty} \cos \left(G_{z} z\right) \cos \left(G_{y} y\right) K_{0} \\
& \times\left(G_{x} \sqrt{y^{2}+z^{2}}\right) d z d y \\
= & \frac{4 \pi}{G_{\|}} \int_{0}^{R} \cos \left(G_{z} z\right) e^{-G_{\|} z} d z,
\end{aligned}
$$

and finally ${ }^{33}$

$$
\widetilde{v}^{2 \mathrm{D}}\left(G_{\|}, G_{z}\right)=\frac{4 \pi}{G^{2}}\left[1+e^{-G_{\|} R} \frac{G_{z}}{G_{\|}} \sin \left(G_{z} R\right)-e^{-G_{\|} R} \cos \left(\left|G_{z}\right| R\right)\right] .
$$

In the limit $R \rightarrow \infty$ the unscreened potential $\frac{4 \pi}{G^{2}}$ is recovered. Similarly to the case of $1 \mathrm{D}$, the limit $G \rightarrow 0$ does not exist, since for $G_{z}=0$, the cutoff has a finite value, while it diverges in the limit $G_{\|} \rightarrow 0$

$$
\widetilde{v}^{2 \mathrm{D}}\left(G_{\|}, G_{z}\right) \sim \frac{1}{G_{\|}^{2}} \text { for } G_{\|}>0, G_{z} \rightarrow 0^{+} .
$$

Up to this point we have not committed to a precise value of the cutoff length $R$. This value has to be chosen, for each dimensionality, in such a way that it avoids the interaction of any two neighbor images of the unit cell in the nonperiodic dimension.

In order to fix the values of $R$ we must choose the size of the supercell. This leads us to step (3) of our procedure. We recall that even once the long-range interaction is cutoff out of some region around each component of the system, this is still not sufficient to avoid the interaction among undesired images. The charge density has to be modified, or, equivalently, the supercell has to be built in such a way that two neighboring densities along every nonperiodic direction do not interact via the cutoff interaction.

It is easy to see how this could happen in the simple case of a 2D square cell of length $L$ : if both $\mathbf{r}$ and $\mathbf{r}^{\prime}$ belong to the cell, then $r, r^{\prime} \leqslant L$, and $\left|\mathbf{r}-\mathbf{r}^{\prime}\right| \leqslant \sqrt{2} L$. If a supercell is built that is smaller than $(1+\sqrt{2}) L$, there could be residual interaction, and the cutoff would no longer lead to the exact removal of the undesired interactions.

Let us call $A_{0}$ the unit cell of the system we are working with, and $\mathcal{A}=\left\{A_{i}, i=-\infty, \ldots, \infty\right\}$ the set of all the cells in the system. If the system is $n \mathrm{D}$ periodic this set only includes the periodic images of $A_{0}$ in the $n$ periodic directions. Let us call $\mathcal{B}$ the set of all the nonphysical images of the system, i.e., those in the nonperiodic directions. Then $\mathcal{A} \cup \mathcal{B}=\mathbb{R}^{3}$. Obviously, if the system is 3D-periodic $\mathcal{A}=\mathbb{R}^{3}$, and $\mathcal{B} \equiv \varnothing$.

In general we want to allow the interaction of the electrons in $A_{0}$ with the electrons in all the cells $A_{i} \in \mathcal{A}$, but not with those $B_{i} \in \mathcal{B}$. To obtain this, we define the supercell $C_{0} \supseteq A_{0}$ such that its length equals the lattice constant of the system in the periodic directions, while some amount of vacuum is added in the nonperiodic directions. The only case for which $C_{0}=A_{0}$ is the 3D-periodic case (see Fig. 1 for a simplified $2 \mathrm{D}$ sketch). The density $\widetilde{n}(\mathbf{r})$ in the cell $C_{0}$ satisfies the conditions

$$
\text { if } \mathbf{r} \in A_{0} \text { then } \widetilde{n}(\mathbf{r})=n(\mathbf{r}) \text {, }
$$




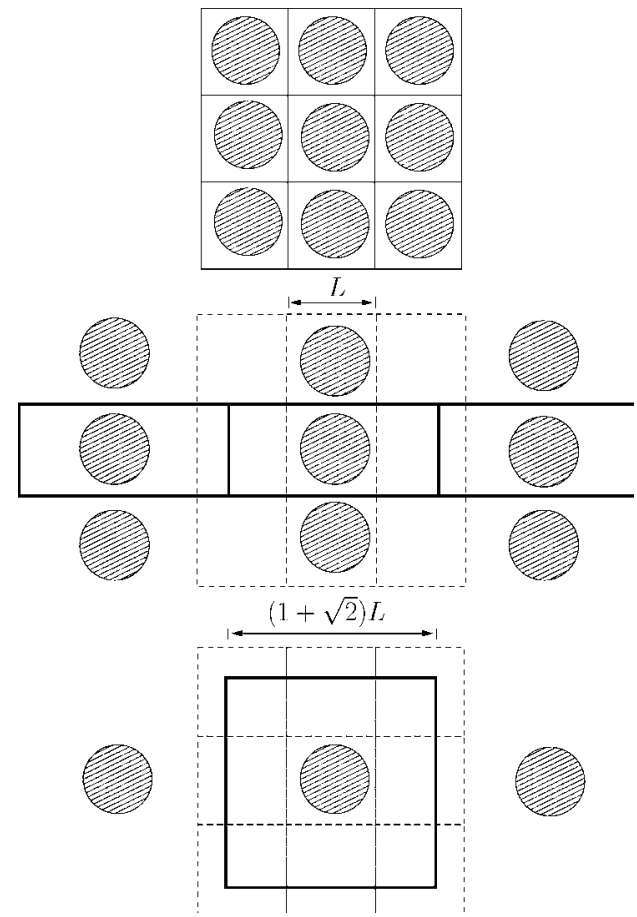

FIG. 1. Schematic description for the supercell construction in a $2 \mathrm{D}$ system. The upper sketch corresponds to the 2D-periodic case (i.e., a 2D bulk crystal). The middle sketch corresponds to a 0Dperiodic system (i.e., a finite $2 \mathrm{D}$ system), and the bottom one to a $1 \mathrm{D}$-periodic (i.e., an isolated chain). In the 0D-periodic case the electrons belonging to different cells do not interact, while in the 1D-periodic a chain does not interact with another, but all the electrons of the chain do interact with each other (see text for details).

$$
\text { if } \mathbf{r} \in C_{0} \text { and } \mathbf{r} \notin A_{0} \text { then } \widetilde{n}(\mathbf{r})=0 .
$$

The size $L_{C}$ of the super cell in the nonperiodic directions depends on the periodic dimensionality of the system. In order to completely avoid any interaction, even in the case the density of the system is not zero at the cell border, for a $3 \mathrm{D}$ system it has to be

$$
\begin{gathered}
L_{C}=(1+\sqrt{3}) L \text { for the finite case, } \\
L_{C}=(1+\sqrt{2}) L \text { for the 1D-periodic case, } \\
L_{C}=2 L \quad \text { for the } 2 \mathrm{D} \text {-periodic case. }
\end{gathered}
$$

Actually, since the required super cell is quite large, a compromise between speed and accuracy can be achieved in the computation, using a parallelepiped super cell with $L_{C}=2 L$, for all cases. This approximation rests on the fact that the charge density is usually contained in a region smaller than the cell in the nonperiodic directions, so that the spurious interactions are, in fact, avoided, even with a smaller cell. Therefore, on the basis of this approximation, we can choose the value of the cutoff length $R$ always as half the smallest primitive vector in the nonperiodic dimension.

Figure 1 schematically illustrates how the supercell is built for a 2D system in all the possible cases, i.e., 2D periodic, 1D periodic, and OD periodic. The charge density of the system is represented by the circles. Straight lines represent (super)cell boundaries, while dashed lines reproduce the 2Dperiodic lattice, and are left in place for reference. The upper sketch corresponds to the 2D-periodic case (i.e., a 2D crystal, with lattice constant $L$ ). No supercell is needed here. The middle sketch corresponds to a 1D-periodic system. The interaction between two different chains is quenched, but it is allowed among all the elements belonging to each chain. The bottom sketch refers to the 0D-periodic case. None of the images can interact with the system in the middle supercell.

\section{CANCELLATION OF THE SINGULARITIES}

The main point in the procedure of eliminating the divergences in all the cases of interest is to observe that our final goal is usually not to obtain the expression of the Hartree potential alone. In fact all the physical quantities depend on the total potential, i.e., on the sum of the electronic and the ionic potential. When this sum is considered we can exploit the fact that each potential is defined up to an arbitrary additive constant, and choose the constants consistently for the two potentials. Since we know in advance that the sum must be finite, we can include, so to speak, all the infinities into these constants, provided that we find a method to separate out the long range part of both potentials on the same footing.

In what follows we show how charge neutrality can be exploited to obtain the exact cancellations when operating with the cutoff expression of Sec. III in Fourier space.

The total potential of the system is built in the following way: we separate out first short and long range contributions to the ionic potential by adding and subtracting a Gaussian charge density $n_{+}(r)=Z \exp \left(-a^{2} r^{2}\right)$. The potential generated by this density is $V_{+}(r)=Z \frac{\operatorname{erf}(a r)}{r}$. The ionic potential is then written as

$$
V(r)=\Delta V(r)-V_{+}(r)
$$

where $a$ is chosen so that $\Delta V(r)$ is localized within a sphere of radius $r_{a}$, smaller than the cell size. The expression of the ionic potential in reciprocal space is

$$
V(G)=\Delta V(G)-V_{+}(G),
$$

where

$$
\begin{gathered}
\Delta V(G)=4 \pi \int_{0}^{+\infty} \frac{r \sin (G r)}{G} \Delta V(r) d r \\
V_{+}(G)=\frac{4 \pi}{G^{2}} \exp \left(-\frac{G^{2}}{4 a^{2}}\right) .
\end{gathered}
$$

The limit for $G=0$ gives a finite contribution from the first term, and a divergent contribution from the second term

$$
\lim _{G \rightarrow 0} \Delta V(G)=4 \pi \int_{0}^{+\infty} r^{2} \Delta V(r) d r
$$




$$
\lim _{G \rightarrow 0} V_{+}(G)=+\infty
$$

The first is the contribution of the localized charge. It is easily computed, since the integrand is zero for $r>r_{a}$. The second term is canceled by the corresponding $G=0$ term in the electronic Hartree potential, due to the charge neutrality of the system. This trivially solves the problem of the divergences in 3D-periodic systems.

Now let us consider a 1D-periodic system. If we take the general convolution in Eq. (4), and we perform the Fourier series expansion along the periodic $x$ direction, we get the Hartree potential expression

$$
\begin{aligned}
V(x, y, z)= & \sum_{G_{x}} \iint_{\Omega} n\left(G_{x}, y^{\prime}, z^{\prime}\right) v\left(G_{x}, y-y^{\prime}, z-z^{\prime}\right) \\
& \times e^{i G_{x} x^{\prime}} d y^{\prime} d z^{\prime} .
\end{aligned}
$$

Invoking the charge neutrality along the chain axis, we have that the difference between electron and ionic densities satisfies

$$
\iint\left[n_{\text {ion }}\left(G_{x}=0, y, z\right)-n_{\mathrm{el}}\left(G_{x}=0, y, z\right)\right] d y d z=0 .
$$

Unfortunately, the cutoff function in Eq. (13) is divergent for $G_{x}=0$. The effective potential therefore reduces to an undetermined $0 \times \infty$ form. However, we can work out an analytical expression for it by defining first a finite length cylindrical cutoff, but later bringing the size of the cylinder to infinity. In this way, as a first step, we get a new cutoff interaction in a finite cylinder of radius $R$, and length $h$, assuming that $h$ is much larger than the cell size in the periodic direction. In this case the modified finite cutoff potential includes a term

$$
\tilde{v}^{1 \mathrm{D}}\left(G_{x}, r, h\right) \propto \ln \left(\frac{h+\sqrt{h^{2}+r^{2}}}{r}\right),
$$

which, in turn gives, for the plane $G_{x}=0$

$$
\begin{aligned}
\widetilde{v}^{1 \mathrm{D}}\left(G_{x}=0, G_{\perp}\right) \approx & -4 \pi \int_{0}^{R} r J_{0}\left(G_{\perp} r\right) \ln (r) d r \\
& +4 \pi R \ln (2 h) \frac{J_{1}\left(G_{\perp} R\right)}{G_{\perp}} .
\end{aligned}
$$

The effective potential is now split into two terms, of which only the second one depends on $h$. The second step is achieved by going to the limit $h \rightarrow+\infty$, to obtain the exact infinite cutoff. By calculating this limit, we notice that only the second term in the right hand side of Eq. (29) diverges. This term is the one that can be dropped due to charge neutrality (in fact it has the same form for the ionic and electronic charge densities). Thus, for the cancellation to be effective in a practical implementation, we have to treat in the same way both the ionic and Hartree Coulomb contributions. The first term on the right hand side of Eq. (29) must always be taken into account, since it affects both the long and the short range part of the cutoff potentials.
Following this procedure, we obtain a considerable computational advantage, over, e.g., the method originally proposed by Spataru et al. ${ }^{35}$ since our cutoff is just an analytical function of the reciprocal space coordinates, and the evaluation of an integral for every value of $G_{x}, G_{\perp}$ is not needed. The cutoff proposed in Ref. 35 is actually a particular case of our cutoff, obtained by using the finite cylinder for all the components of the $\mathbf{G}$ vectors: in this case the quadrature in Eq. (29) has to be evaluated for each $G_{x}, G_{y}$, and $G_{z}$, and a convergence study in $h$ is mandatory (see discussion in Sec. V B, and Fig. 5).

In the $1 \mathrm{D}$-periodic case, the $G=0$ value is now well defined, and it turns out to be $\lim _{G_{\perp} \rightarrow 0} \tilde{v}\left(G_{x}, G_{\perp}\right)$

$$
\tilde{v}^{1 \mathrm{D}}\left(G_{x}=0, G_{\perp}=0\right)=-\pi R^{2}[2 \ln (R)-1] .
$$

The analogous result for the 2D-periodic cutoff is obtained by imposing finite cutoff sizes $h_{x}=\alpha h_{y}=h$ (much larger than the cell size), in the periodic directions $x$ and $y$, and dropping the $h$ dependent part before passing to the limit $h \rightarrow+\infty$. The constant $\alpha$ is the ratio $\frac{L_{x}}{L_{y}}$ between the in-plane lattice vectors

$$
\begin{aligned}
\widetilde{v}^{2 \mathrm{D}}\left(G_{\|}\right. & \left.=0, G_{z}\right) \\
\approx & \frac{4 \pi}{G_{z}^{2}}\left[1-\cos \left(G_{z} R\right)-G_{z} R \sin \left(G_{z} R\right)\right] \\
& +8 h \ln \left(\frac{\left(\alpha+\sqrt{1+\alpha^{2}}\right)\left(1+\sqrt{1+\alpha^{2}}\right)}{\alpha}\right) \frac{\sin \left(G_{z} R\right)}{G_{z}} .
\end{aligned}
$$

The $G=0$ value is

$$
\widetilde{v}^{2 \mathrm{D}}\left(G_{\|}=0, G_{z}=0\right)=-2 \pi R^{2} .
$$

To summarize, the divergences can be cancelled also in 1D-periodic and 2D-periodic systems provided that (1) we apply the cutoff function to both the ionic and the electronic potentials, (2) we separate out the infinite contribution as shown above, and (3) we properly account for the short range contributions as stated in Table I. The analytical results of the present work are condensed in Table I: all possible values for the cutoff functions are listed there as a quick reference for the reader.

\section{RESULTS}

The scheme illustrated above has been implemented both in the real space time-dependent DFT code OCTOPUS, ${ }^{31}$ and in the plane wave many-body-perturbation-theory (MBPT) code SELF. ${ }^{36}$ The tests have been performed on the prototypical cases of infinite chains of atoms along the $x$ axis. We compare between the 3D-periodic calculation (physically corresponding to a crystal of chains), and the 1D-periodic case (corresponding to the isolated chain) both in the usual supercell approach, and within our exact screening method. The discussion for the 2D cases is similar as for the 1D case, while results for the finite systems have already been reported in the literature. ${ }^{26,30}$ We addressed different properties to see the impact of the cutoff at each level of calculation, 
TABLE I. Reference table summarizing the results of the cutoff work for charge-neutral systems: finite systems (OD), one-dimensional systems (1D) and two-dimensional systems (2D). The complete reciprocal space expression of the Hartree potential is provided. For the 1D case, $R$ stands for the radius of the cylindrical cutoff whereas in the 0D case it is the radius of the spherical cutoff. In 2D $R$ stands for half the thickness of the slab cutoff (see text for details).

\begin{tabular}{ccc}
\hline \hline$G$ & & 0D-periodic $\widetilde{v}^{0 D}(G)=$ \\
$\neq 0$ & $\left(4 \pi / G^{2}\right)[1-\cos (G R)]$ \\
0 & & $2 \pi R^{2}$ \\
& & \\
$G_{x}$ & $G_{\perp}$ & $1 \mathrm{D}$-periodic $\widetilde{v}^{1 D}\left(G_{x}, G_{\perp}\right)=$ \\
$\neq 0$ & any & $\left(4 \pi / G^{2}\right)\left[1+G_{\perp} R J_{1}\left(G_{\perp} R\right) K_{0}\left(\left|G_{x}\right| R\right)\right.$ \\
& $\left.\quad-\left|G_{x}\right| R J_{0}\left(G_{\perp} R\right) K_{1}\left(\left|G_{x}\right| R\right)\right]$ \\
0 & $\neq 0$ & $-4 \pi \int_{0}^{R} r J_{0}\left(G_{\perp} r\right) \ln (r) \mathrm{d} r$ \\
0 & 0 & $-\pi R^{2}[2 \ln (R)-1]$ \\
& $\quad$ & $\left(4 \pi / G^{2}\right)\left\{1+e^{-G_{\|} R}\left[\left(G_{z} / G_{\|}\right) \sin \left(G_{z} R\right)-\cos \left(G_{z} R\right)\right]\right\}$ \\
$G_{\|}$ & $G_{z}$ & $2 \mathrm{D}-$ periodic $\widetilde{v}^{2 D}\left(G_{\|}, G_{z}\right)=$ \\
$\neq 0$ & any & $\left(4 \pi / G_{z}^{2}\right)\left[1-\cos \left(G_{z} R\right)-G_{z} R \sin \left(G_{z} R\right)\right]$ \\
0 & $\neq 0$ & $-2 \pi R^{2}$ \\
0 & 0 & \\
\hline \hline
\end{tabular}

from the ground state to excited state and quasiparticle dynamics.

\section{A. Ground-state calculations}

All the calculations have been performed with the realspace implementation of DFT in the ${ }^{31}$ OCTOPUS code. We have used nonlocal norm-conserving pseudopotentials ${ }^{37}$ to describe the electron-ion interaction and the local-density approximation $^{38}$ (LDA) to describe exchange-correlation effects. The particular choice of exchange-correlation or ionic pseudopotential is irrelevant here as we want to assess the impact of the Coulomb cutoff and this is independent of those quantities. The density and the wave functions are represented in real space using a cubic regular mesh. The spacing between the grid points is 0.38 a.u. (0.2 $\AA$ ) for $\mathrm{Na}$, which is the largest spacing that allows us to represent the corresponding pseudopotentials.

In this case, the trace of the interaction of neighboring chains in the $y$ and $z$ directions is the dispersion of the bands in the corresponding direction of the Brillouin zone. However it is known that, if the supercell is large enough, the bands along the $\Gamma-X$ direction are unchanged. This apparently contradicts the fact that the radial ionic potential for a wire [that asymptotically goes like $\ln (r)$ as a function of the distance $r$ from the axis of the wire] is completely different from the crystal potential.

We can resolve the contradiction by performing a cutoff calculation. In fact, the overall effect of the interaction of neighboring chains on the ground-state occupied states turns out to be canceled by the Hartree potential, i.e., by the electron screening of the ionic potential, but two different scenarios become clear as soon as the proper cutoff is used.
Figure 2 (top) shows the ionic potential, as well as the Hartree potential and their sum for a $\mathrm{Na}$ atom in a parallelepiped supercell with side lengths of 7.6, 18.8, and 18.8 a.u. $(4 \times 10 \times 10 \AA)$ respectively in the $x, y$, and $z$ directions. No cutoff is used here. The ionic potential behaves roughly like $\frac{1}{r}$ in the area not too close to the nucleus (where the pseudopotential dominates). The total potential, on the other hand, falls off rapidly to an almost constant value at around 6 a.u. from the nuclear position, by effect of the electron screening.

Figure 2 (bottom) shows the results when the cutoff is applied (the radius of the cylinder is $R=18.8$ a.u. such that there is zero interaction between cells). The ionic potential now behaves as it is expected to for the potential of a chain, i.e., diverges logarithmically, and is clearly different from the latter case. Nevertheless the sum of the ionic and Hartree potential is basically the same as for the 3D-periodic system.

The two band structures are then expected, and are found to be the same, confirming that, as far as static calculations are concerned, the supercell approximation is good, provided that the supercell is large enough. In static calculations, then, the use of our cutoff only has the effect of allowing us to eventually use a smaller supercell, which clearly saves computational time. In the case of the Na-chain, a full 3D calculation would need a cell size of 38 a.u. (20 $\AA$ ) whereas the cutoff calculation would give the same result with a cell size of 19 a.u. (10 $\AA$ ), and $k$ sampling along the chain axis only. Naturally, when more delocalized states are considered, like higher energy unoccupied states, larger differences are observed with respect to the supercell calculation.

In Fig. 3 we show the effect of the cutoff on the occupied and unoccupied states. As expected, the occupied states are not affected by the use of the cutoff, since the density of the system within the cutoff radius is unchanged, and the corresponding band is the same as it is found for an ordinary $3 \mathrm{D}$ supercell calculation with the same cell size. However there is a clear effect on the bands corresponding to unoccupied states, and the effect is larger the higher the energy of the states. In fact the high energy states, and the states in the continuum are more delocalized. Therefore the effect of the boundary conditions is more important. We obtain the same result for a $\mathrm{Si}$ chain.

To summarize: for the static case, we have computed the band structure of a single chain in two cases: with no cutoff in a wide supercell, and with cutoff in a much smaller supercell, and without $k$ points sampling in the direction perpendicular to the chain axis. We might think as the wide supercell calculation as a reference (provided a rigid shift in the energy values is allowed), even though the convergence to the actual physical values of the isolated system is well known to be very slow. We obviously must keep the periodic boundary conditions along the axis, but in the other directions we have applied zero boundary conditions. In this situation the comparison between the two band structures makes sense only up to some energy, which, in turn, depends on the cell size. Above that energy box states appear in the cutoff calculation, and the two band structures start differing. Fortunately, since the ground-state properties of the system depend uniquely on its density, it is sufficient to obtain an agreement in all the occupied states. The wider the supercell 


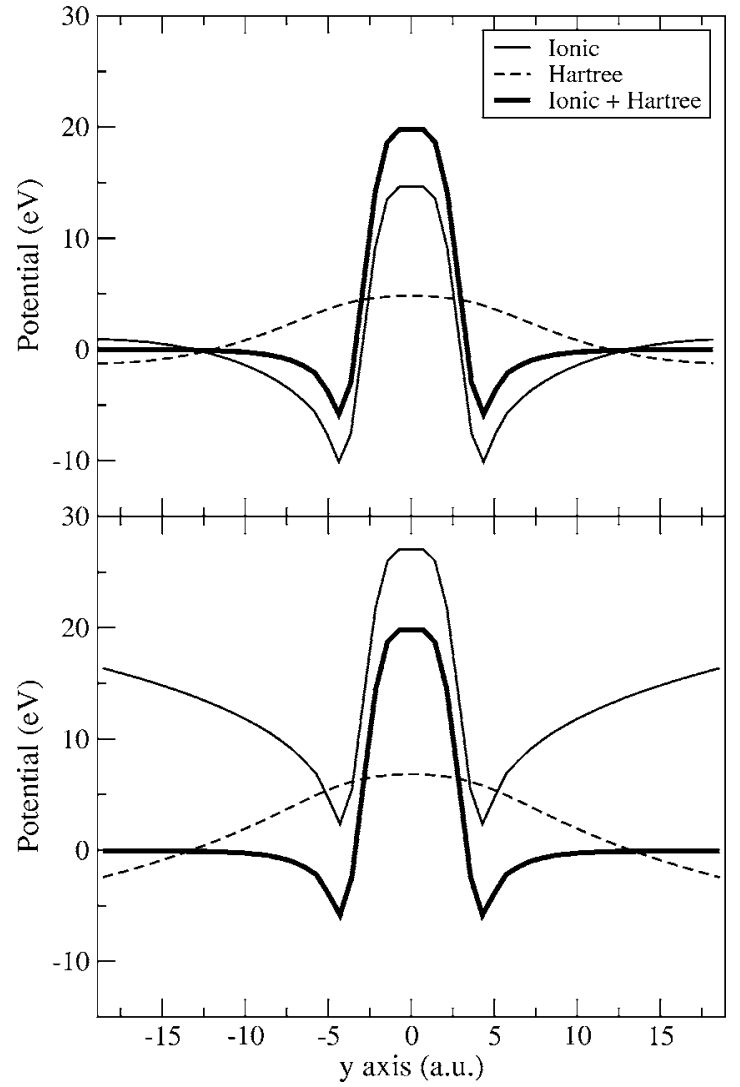

FIG. 2. Calculated total and ionic and Hartree potentials for a 3D-periodic (top) and 1D-periodic (bottom) $\mathrm{Na}$ chain.

in the nonperiodic direction, the higher in energy becomes the agreement between the two calculations.

As an example of the same situation in the 2D-periodic case we have computed the band structure of a sheet of $\mathrm{Na}$ atoms, both in the periodic supercell approximation, and using the cutoff scheme. The results are shown in Fig. 4. The discussion and the conclusions are analogous to the 1Dperiodic case: a band structure calculation with planar cutoff in a $7.6 \times 7.6 \times 18.8$ a.u. $(4 \times 4 \times 10 \AA)$ wide cell gives the same lowest energy bands as a calculation with no cutoff in a $7.6 \times 7.6 \times 38$ a.u. supercell. Bounding box effects modify the unoccupied states.

As a concluding remark for this section, we stress that the only purpose of the cutoff technique is to remove the spurious effect of the Coulomb interaction in the nonperiodic directions of a system, independently of the nature of the surrounding medium. In order to focus on this effect we have tested the cutoff using zero boundary conditions in the nonperiodic directions. On the other hand the combined use of a cutoff together with suitably defined boundary conditions would allow one to address a larger set of cases, such as the case of defects in bulk solids away from the surface, etc. The derivation of the boundary conditions that suit each class of problems, however, is not part of the cutoff problem, and it is not addressed in what follows.

\section{B. Static polarizability}

After the successful analysis of the ground state properties with the cutoff scheme, we have applied the modified Cou-

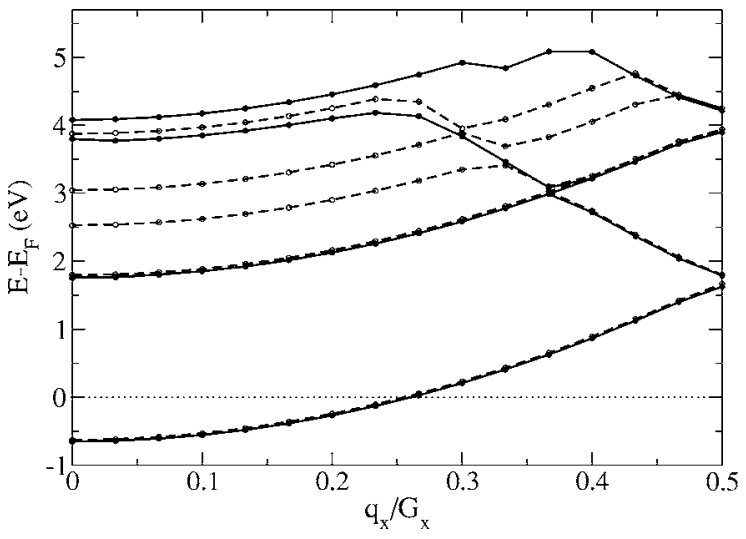

FIG. 3. Effect of the cutoff in a $\mathrm{Na}$ linear chain in a supercell size of $7.5 \times 19 \times 19$ a.u. The bands obtained with an ordinary supercell calculation with no cutoff (dashed lines) are compared to the bands obtained applying the 1D cylindrical cutoff (solid line). As is explained in the text, only the unoccupied levels are affected by the cutoff.

lomb potential to calculate the static polarizability of an infinite chain in the random phase approximation (RPA). As a test case, we have considered a chain made of hydrogen atoms, two atoms per cell at a distance of 2 a.u. (1.06 $\AA$ ). The lattice parameter was 4.5 a.u. $(2.4 \AA)$. For this system we have also calculated excited-state properties in manybody perturbation theory, in particular, the quasiparticle gap in Hedin's GW approximation ${ }^{6}$ and the optical absorption spectra in the Bethe-Salpeter framework ${ }^{7,39}$ (see sections below). All these calculations have been performed in the code SELF. ${ }^{36}$ The polarizability for the monomer, which is a finite system, in the RPA including local field effects is defined as

$$
\alpha=-\lim _{\mathbf{q} \rightarrow 0} \frac{1}{q^{2}} \chi_{00}(\mathbf{q}) \frac{\Omega}{4 \pi},
$$

where $\chi_{G G^{\prime}}(q)$ is the interacting polarization function that is a solution of the Dyson-like equation

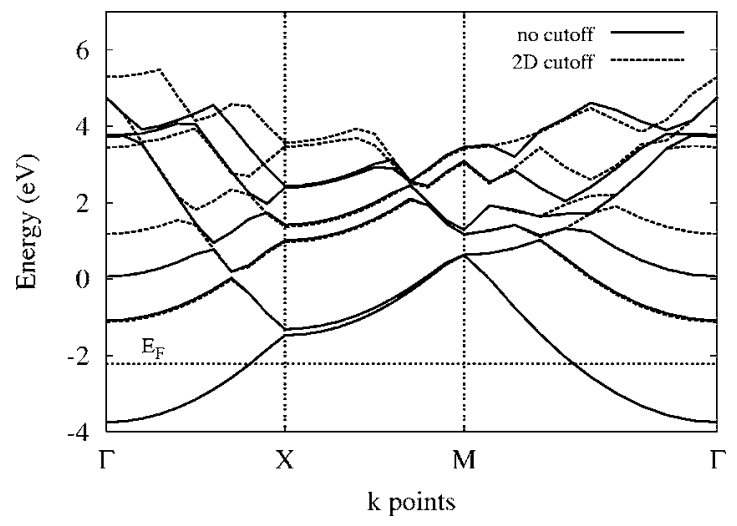

FIG. 4. Band structure of a planar sheet of $\mathrm{Na}$ atoms in a supercell size of $7.5 \times 7.5 \times 19$ a.u. The bands with and without cutoff are identical for all the occupied states. The unoccupied states are influenced by different boundary conditions. 


$$
\chi_{G G^{\prime}}(\mathbf{q})=\chi_{G G^{\prime}}^{0}(\mathbf{q})+\sum_{G^{\prime \prime}} \chi_{G G^{\prime \prime}}^{0}(\mathbf{q}) v\left(\mathbf{q}+\mathbf{G}^{\prime \prime}\right) \chi_{G^{\prime \prime} G^{\prime}}(\mathbf{q})
$$

and $\chi^{0}$ is the noninteracting polarization function obtained by the Adler-Wiser expression. ${ }^{40} v(\mathbf{q}+\mathbf{G})$ are the Fourier components of the Coulomb interaction. Note that the expression for $\alpha$ in Eq. (33) is also valid for calculations in finite systems, in the supercell approximation, and the dependence on the wave vector $q$ is due to the representation in reciprocal space.

In the top panel of Fig. 5, we compare the values of the calculated polarizability $\alpha$ for different supercell sizes. $\alpha$ is calculated both using the bare Coulomb $v(\mathbf{q}+\mathbf{G})=\frac{4 \pi}{|\mathbf{q}+\mathbf{G}|^{2}}$ and the modified cutoff potential of Eq. (13) (the radius of the cutoff is always set to half the interchain distance). The lattice constant along the chain axis is kept fixed. Using the cutoff, the static polarizability already converges to the asymptotic value with an interchain distance of 25 a.u. (13.2 $\AA$ ). Without the cutoff the convergence is much slower, and the exact value is approximated to the same accuracy for much larger cell sizes (beyond the calculations shown in the top of Fig. 5).

We must stress the fact that the treatment of the divergences in this case is different with respect to the case of the Hartree and ionic potential cancellation for ground-state calculations (i.e., charge neutrality). In fact, while in the calculation of the Hartree and ionic potential the divergent terms are simply dropped by virtue of the neutralizing positive background, here the $h$ dependence in Eq. (29) can be removed only for the head component by virtue of the vanishing limit $\lim _{\mathbf{q} \rightarrow 0} \chi_{00}^{0}(\mathbf{q})=0$, while for the other $G_{x}=0$ components we have to resort to the expression of the finite cylindrical cutoff as in Eq. (29).

A finite version of the 1D cutoff has been recently applied to nanotube calculations. ${ }^{35,41}$ This cutoff was obtained by numerically truncating the Coulomb interaction along the axis of the nanotube, in addition to the radial truncation. Therefore, the effective interaction is limited to a finite cylinder, whose size can be up to a hundred times the unit cell size, depending on the density of the $k$ point sampling along the axis. ${ }^{5}$ The cutoff axial length has to be larger than the expected bound exciton length.

In the bottom part of Fig. 5 we compare the results obtained with our analytical cutoff [Eq. (13)] with its finite special case proposed in Ref. 35 . We observe that the value of the static polarizability calculated with the finite cutoff oscillates around an asymptotic value, for increasing axial cutoff lengths. The asymptotic value exactly coincides with the value obtained with our cutoff. We stress the fact that we also resort to the finite form of the cutoff only to handle the diverging of components of the potential. Thus we note that there is a clear numerical advantage in using our expression, since the cutoff is analytical for all values except at $G_{x}=0$, and the corresponding quadrature has to be numerically evaluated for these points only. In the inset of the bottom part of Fig. 5, the convergence of the polarizability obtained with

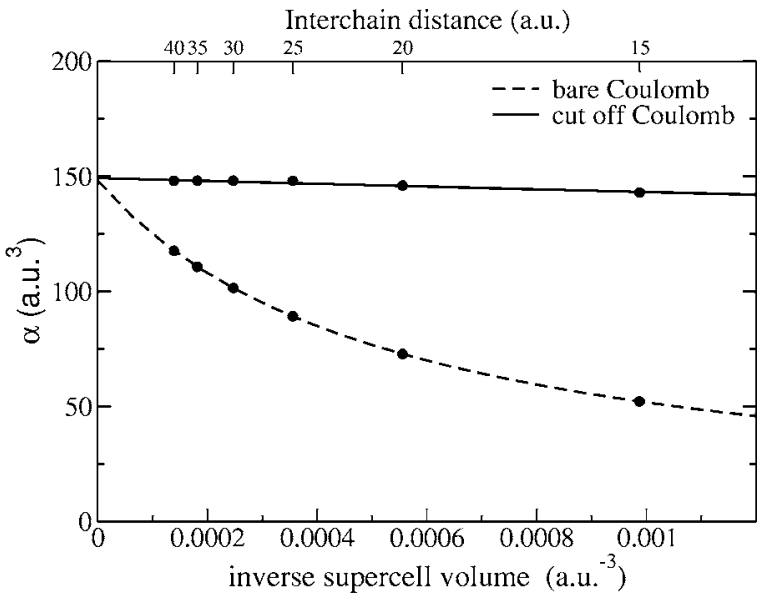

(b)

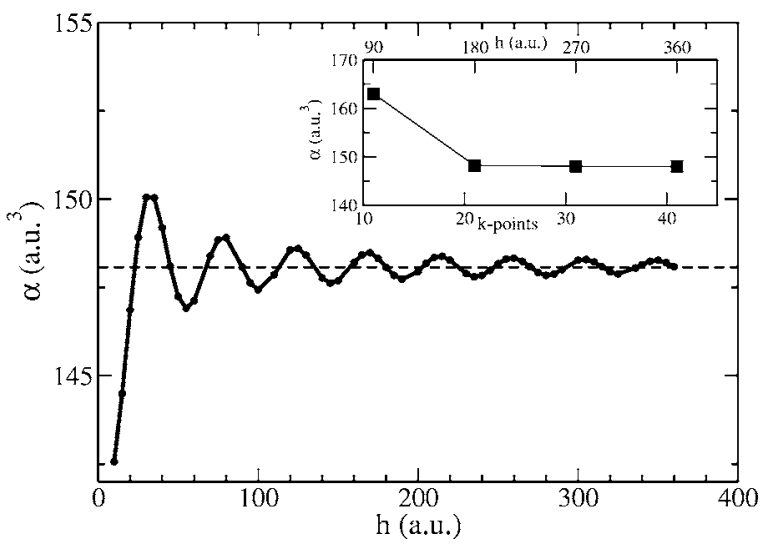

FIG. 5. Top: Polarizability per unit cell of an $\mathrm{H}_{2}$ chain in RPA as a function of the inverse supercell volume. The solid line extrapolates the values obtained with the cutoff potential, while the dashed lines extrapolates the values obtained with the bare Coulomb potential. The cutoff radius is 8.0 a.u. $(4.2 \AA)$. The interchain distance is indicated in the top axis. Bottom: Polarizability of the $\mathrm{H}_{2}$ chain calculated with the finite cutoff potential of Ref. 35 . In abscissa different values of the cutoff length along the chain axis. The dashed straight line indicates the value obtained with the cutoff of Eq. (13). In the inset we show the convergence of the polarizability with respect to the $k$ points sampling along the chain axis obtained with the cutoff of Eq. (13). In the upper axis it is indicated the maximum allowed length $h$ for each $k$ point sampling used in the calculation of the $G_{x}=0$ components by Eq. (29).

our cutoff with respect to the $k$ point sampling is also shown. The sampling is unidimensional along the axial direction. Observe that the calculation using our cutoff is already converged for a sampling of $20 k$ points. In the upper axis, we also indicate the corresponding maximum allowed value of the finite cutoff length in the axial direction that has been used to calculate the $G_{x}=0$ components.

Finite-size effects turn out to be also relevant for manybody perturbation theory calculations. For the same test system (linear $\mathrm{H}_{2}$ chain), in the next two sections, we consider the performance of our cutoff potential for the calculation of the quasiparticle energies in ${ }^{6} \mathrm{GW}$ approximation and of the absorption spectra in the Bethe-Salpeter framework. ${ }^{7,39}$ 


\section{Quasiparticles in the $G W$ approximation}

In the GW approximation, the nonlocal energy-dependent electronic self-energy $\Sigma$ plays a role similar to that of the exchange-correlation potential of DFT. $\Sigma$ is approximated by the convolution of the one-electron Green's function and the dynamically screened Coulomb interaction $W$. We first calculate the ground state electronic properties using the DFT code ABINIT. ${ }^{42}$ These calculation are performed in $^{38}$ LDA with pseudopotentials. ${ }^{37}$ An energy cutoff of $816 \mathrm{eV}$ (30 hartree) has been used to get converged results. The LDA eigenvalues and eigenfunctions are then used to construct the RPA screened Coulomb interaction $W$, and the GW self-energy. The inverse dielectric matrix $\epsilon_{G, G^{\prime}}^{-1}$ has been calculated using the plasmon-pole approximation ${ }^{43}$ and the quasiparticle energies have been calculated to first order in $\Sigma$ $-V_{x c}{ }^{44}$ Dividing the self-energy into an exchange part $\Sigma_{x}$ and a correlation part $\Sigma_{c}\left(\left\langle\phi_{j}^{D F T}|\Sigma| \phi_{i}^{D F T}\right\rangle=\left\langle\phi_{j}^{D F T}\left|\Sigma_{x}\right| \phi_{i}^{D F T}\right\rangle\right.$ $\left.+\left\langle\phi_{j}^{D F T}\left|\Sigma_{c}\right| \phi_{i}^{D F T}\right\rangle\right)$, we get the following representation for the self-energy in a plane-wave basis set

$$
\begin{aligned}
\left\langle n \mathbf{k}\left|\Sigma_{x}\left(\mathbf{r}_{1}, \mathbf{r}_{2}\right)\right| n^{\prime} \mathbf{k}^{\prime}\right\rangle= & -\sum_{n_{1}} \int_{B z} \frac{d^{3} \mathbf{q}}{(2 \pi)^{3}} \sum_{G} v(\mathbf{q}+\mathbf{G}) \\
& \times \rho_{n n_{1}}(\mathbf{q}, \mathbf{G}) \rho_{n^{\prime} n_{1}}^{\star}(\mathbf{q}, \mathbf{G}) f_{n_{1} \mathbf{k}_{1}},
\end{aligned}
$$

and

$$
\begin{aligned}
\langle n \mathbf{k}| \Sigma_{c} & \left(\mathbf{r}_{1}, \mathbf{r}_{2}, \omega\right)\left|n^{\prime} \mathbf{k}^{\prime}\right\rangle \\
= & \frac{1}{2} \sum_{n_{1}} \int_{B z} \frac{d^{3} \mathbf{q}}{(2 \pi)^{3}}\left\{\sum_{\mathbf{G G}^{\prime}} v\left(\mathbf{q}+\mathbf{G}^{\prime}\right) \rho_{n n_{1}}(\mathbf{q}, \mathbf{G})\right. \\
& \times \rho_{n^{\prime} n_{1}}^{\star}\left(\mathbf{q}, \mathbf{G}^{\prime}\right) \int \frac{d \omega^{\prime}}{2 \pi} \epsilon_{\mathbf{G G}}^{-1}\left(\mathbf{q}, \omega^{\prime}\right) \\
& \left.\times\left[\frac{f_{n 1(\mathbf{k}-\mathbf{q})}}{\omega-\omega^{\prime}-\epsilon_{n 1(\mathbf{k}-\mathbf{q})}^{L D A}-i \delta}+\frac{1-f_{n 1(\mathbf{k}-\mathbf{q})}}{\omega-\omega^{\prime}-\epsilon_{n 1(\mathbf{k}-\mathbf{q})}^{L D A}+i \delta}\right]\right\},
\end{aligned}
$$

where $\rho_{n n_{1}}(\mathbf{q}+\mathbf{G})=\left\langle n \mathbf{k}\left|e^{i(\mathbf{q}+\mathbf{G}) \cdot \mathbf{r}_{1}}\right| n_{1} \mathbf{k}_{1}\right\rangle$ and the integral in the frequency domain in Eq. (36) has been analytically solved considering the dielectric matrix in the plasmon pole mode: $\left(\epsilon_{\mathbf{G}, \mathbf{G}^{\prime}}^{-1}(\omega)=\delta_{\mathbf{G}, \mathbf{G}^{\prime}}+\Omega_{\mathbf{G}, \mathbf{G}^{\prime}} /\left(\omega^{2}-\tilde{\omega}_{G, G^{\prime}}^{2}\right)\right)$.

In order to eliminate the spurious interaction between different supercells, leaving the bare Coulomb interaction unchanged along the chain direction, we simply introduce the expression of Eq. (13) in the construction of $\Sigma_{x}$ and $\Sigma_{c}$, and also in the calculation of $\epsilon_{G G^{\prime}}^{-1}$. As in the calculation of the static polarizability, the divergences appearing in the components $\left(G_{x}=0\right)$ cannot be fully removed and for such components we resort to the finite version of the cutoff potential Eq. (29).

In Fig. 6 we calculate the convergence of the quasiparticle gap at the $X$ point for different supercell sizes in the $\mathrm{GW}$ approximation. A cutoff radius of 8.0 a.u. has been used. When the cutoff potential is used, $60 k$ points in the axis direction has been necessary to get converged results. In the inset of Fig. 6, we show the behavior of the quasiparticle gap as a function of the cutoff radius. We observe that for $R_{c}$ $>6$ a.u. (3.17 $\AA$ ) a plateau is reached, and, for $R_{c}>12$ a.u., a small oscillation appears due to interaction between the tails of the charge density of the system with its image in the neighboring cell. Unlike the DFT-LDA, calculation for neutral systems, where the supercell approximation turns out to be good, as we have discussed above, we can see that the convergence of the GW quasiparticle correction turns out to be extremely slow with respect to the size of the supercell and huge supercells are needed in order to get converged results. This is due to the fact that in the GW calculation the addition of an electron (or a hole) to the system induces charge oscillation in the periodic images as well. It is important to note that the slow convergence is caused by the correlation part of the self-energy [Eq. (36)], while the exchange part is rapidly convergent with respect to the cell size. The use of the cutoff Coulomb potential improves the convergence drastically as is evident from Fig. 6. Note that even at 38 a.u., the interchain distance the GW gap is underestimated by about $0.5 \mathrm{eV}$. A similar trend (but with smaller variations) has been found by Onida et al. ${ }^{32}$ for a finite system (Sodium Tetramer) using the cutoff potential of Eq. (11). Clearly, there is a strong dimensionality dependence of the selfenergy correction. The nonmonotonic behavior versus dimensionality of the self-energy correction has also been pointed out in Ref. 45 where the gap correction was shown to have a strong component of the surface polarization.

\section{Exciton binding energy: Bethe-Salpeter equation}

Starting from the quasiparticle energies, we have calculated the optical absorption spectra including electron-hole interactions calculated with the Bethe-Salpeter equation. ${ }^{39}$ The basis set used to describe the exciton state is composed of product states of the occupied and unoccupied LDA single particle states and the coupled electron-hole excited states $|S\rangle=\sum_{c v \mathbf{k}} A_{c v \mathbf{k}} a_{c \mathbf{k}}^{\dagger} a_{v \mathbf{k}}|0\rangle$, where $|0\rangle$ is the ground state of the system. $A_{c v \mathbf{k}}$ is the probability amplitude of finding an excited electron in the state $(c \mathbf{k})$ and a hole in $(v \mathbf{k})$. It satisfies the equation

$$
\left(\epsilon_{c \mathbf{k}}^{Q P}-\epsilon_{v \mathbf{k}}^{Q P}\right) A_{v c \mathbf{k}}+\sum_{v c \mathbf{k}, v^{\prime} c^{\prime} \mathbf{k}^{\prime}} K_{v c \mathbf{k}, v^{\prime} c^{\prime} \mathbf{k}^{\prime}} A_{v^{\prime} c^{\prime} \mathbf{k}^{\prime}}=E_{S} A_{v c \mathbf{k}} .
$$

$E_{S}$ is the excitation energy of the state $|S\rangle$ and $K$ the interaction kernel that includes an unscreened exchange repulsive term $K^{\text {Exch }}$ and a screened electron-hole interaction $K^{\text {dir }}$ (direct term). In the plane-wave basis such terms read

$$
K_{\left(v c \mathbf{k}, v^{\prime} c^{\prime} \mathbf{k}^{\prime}\right)}^{E x c h}=\frac{2}{\Omega} \sum_{\mathbf{G} \neq 0} v(\mathbf{G})\left\langle c \mathbf{k}\left|e^{i \mathbf{G} \cdot \mathbf{r}}\right| v \mathbf{k}\right\rangle\left\langle v^{\prime} \mathbf{k}^{\prime}\left|e^{-i \mathbf{G}^{\prime} \cdot \mathbf{r}}\right| c^{\prime} \mathbf{k}^{\prime}\right\rangle
$$

$$
\begin{aligned}
K_{\left(v c \mathbf{k}, v^{\prime} c^{\prime} \mathbf{k}^{\prime}\right)}^{d i r}= & \frac{1}{\Omega} \sum_{\mathbf{G}, \mathbf{G}^{\prime}} v(\mathbf{q}+\mathbf{G}) \boldsymbol{\epsilon}_{\mathbf{G G}^{\prime}}^{-1}(\mathbf{q})\left\langle c \mathbf{k}\left|e^{i(\mathbf{q}+\mathbf{G}) \cdot \mathbf{r}}\right| c^{\prime} \mathbf{k}^{\prime}\right\rangle \\
& \times\left\langle v^{\prime} \mathbf{k}^{\prime}\left|e^{-i\left(\mathbf{q}+\mathbf{G}^{\prime}\right) \cdot \mathbf{r}}\right| v \mathbf{k}\right\rangle \delta_{\mathbf{q} \cdot \mathbf{k}-\mathbf{k}^{\prime}}
\end{aligned}
$$




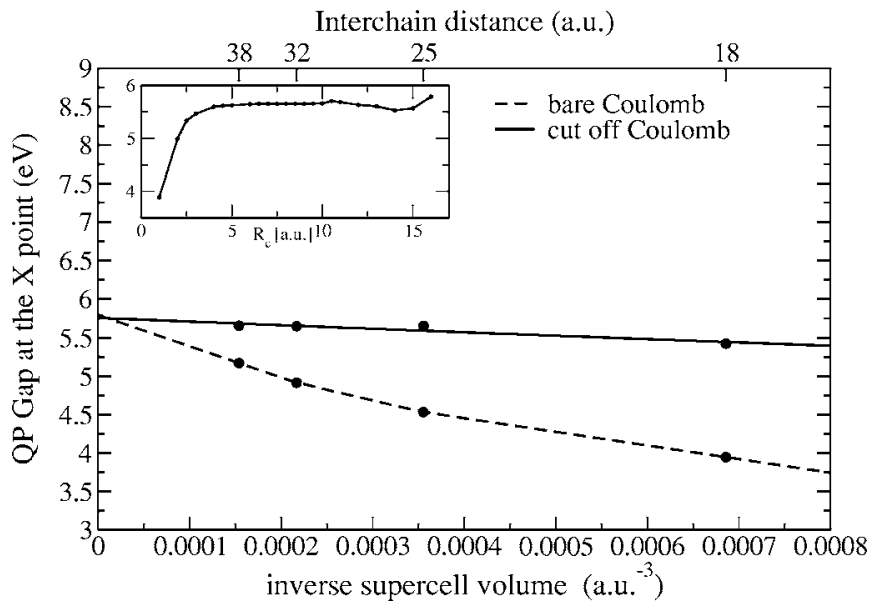

FIG. 6. Convergence of the GW quasiparticle gap for the $H_{2}$ chain as a function of the inverse of the cell size, using the bare Coulomb potential (dashed line) and the cutoff potential (solid line). In the inset the behavior of the GW quasiparticle gap as a function of the value of the cutoff radius for a supercell with inter-chain distance of 32 a.u. (17 $\AA$ ) is shown. The plateau obtained around a radius of 8 a.u. (i.e., one fourth of the supercell size) corresponds to the situation in which the radial images of chains no longer mutually interact, and the calculation is converged. Increasing the radius above approximately 12 a.u. the interaction is back and produces oscillations in the value of the gap.

The screened potential has been treated in static RPA approximation (dynamical effects in the screening have been neglected as it is usually done in recent Bethe-Salpeter calculations ${ }^{39}$ ). The quasiparticle energies in the diagonal part of the Hamiltonian in Eq. (37) are obtained by applying a scissor operator to the LDA energies. This is because in the studied test case the main difference between the quasiparticle and LDA band structure consists of a rigid energy shift of energy bands. From the solution of the Bethe-Salpeter equation [Eq. (37)], it is possible to calculate the macroscopic dielectric function. In particular, the imaginary part reads

$$
\epsilon_{2}(\omega)=\frac{1}{\Omega} \frac{4 \pi^{2} e^{2}}{\omega^{2}} \sum_{S}\left|\sum_{v c \mathbf{k}} A_{v c \mathbf{k}}^{S}\langle v \mathbf{k}|\boldsymbol{\lambda} \cdot \boldsymbol{\nu}| c \mathbf{k}\rangle\right|^{2} \delta\left(E_{S}-\hbar \omega\right)
$$

where the summation runs over all the vertical excitations from the ground state $|0\rangle$ to the excited state $|S\rangle . E_{S}$ is the corresponding excitation energy, $\boldsymbol{\nu}$ is the velocity operator and $\boldsymbol{\lambda}$ is the polarization vector. As in the case of the GW calculation, in order to isolate the chain, we substitute the cutoff potential of Eq. (13) both in the exchange term Eq. (38) and in the direct term of the Bethe-Salpeter equation, Eq. (39), as well as for the RPA dielectric matrix in Eq. (39).

In the top part of Fig. 7, we show the calculated spectra for different cell sizes together with the noninteracting spectrum, and the spectrum obtained using the cutoff Coulomb potential for an interchain distance larger than 20 a.u. (10.6 $\AA$ ) and cylindrical cutoff radius of 8 a.u. The scissor operator applied in this calculation is the same for all the volumes and correspond to the converged GW gap. (a)

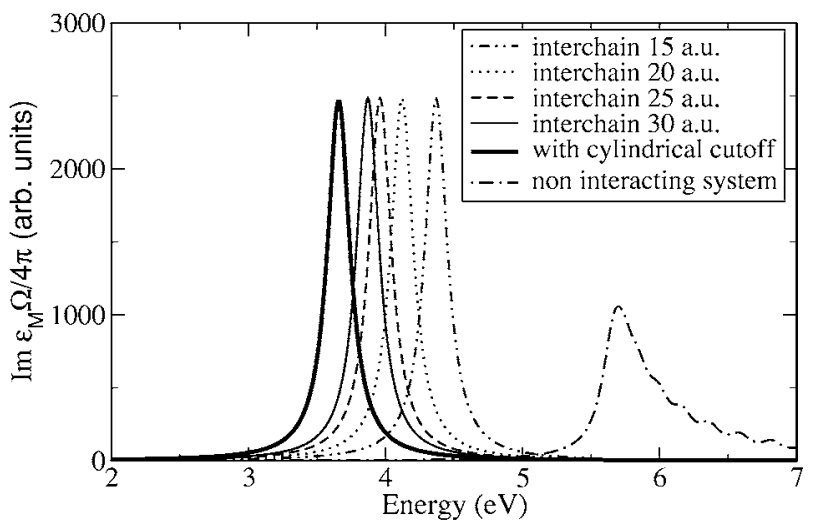

(b)

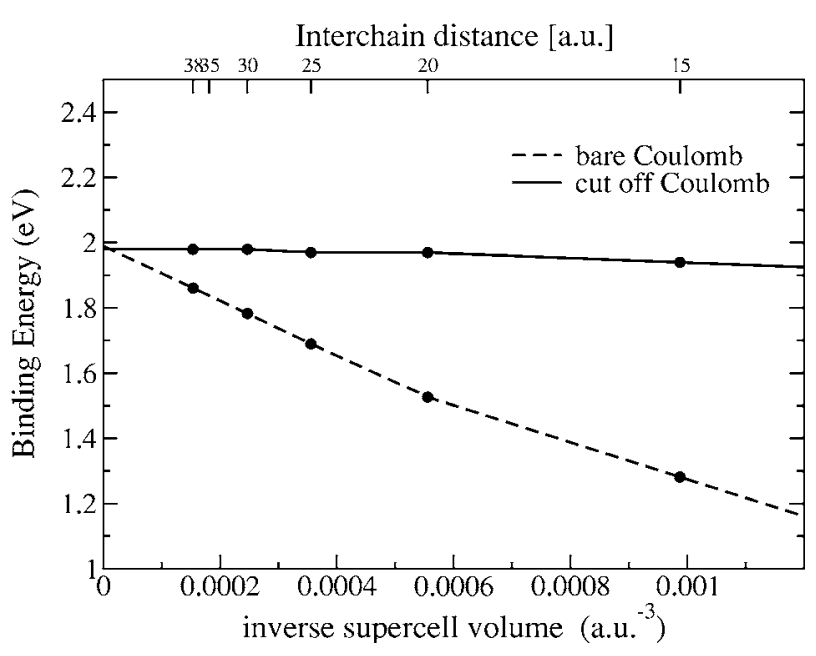

FIG. 7. Top: Photo absorption cross section of a linear chain of $H_{2}$ for different supercell volumes. In the legend the interchain distances corresponding to each volume are indicated. The intensity has been normalized to the volume of the supercell. The noninteracting absorption spectra and the spectra obtained with the cutoff potential are also included. Bottom: exciton binding energy vs supercell volume calculated using the cut off potential (solid line), and the bare Coulomb potential (dashed line).

As it is known, the electron-hole interaction modifies both the shape and the energy of the main absorption peak. This effect is related to the the slow evolution of the polarizability per $\mathrm{H}_{2}$ unit. $^{46}$ Furthermore, the present results clearly illustrate that the spectrum calculated without the cutoff slowly converges towards the exact result. This is highlighted in the bottom panel of Fig. 7 where we show the dependence of the exciton binding energy on the supercell volume, the binding energy being defined as the energy difference between the excitonic peak and the optical gap. We observe that the effect of the interchain interaction consists in reducing the binding energy with respect to its value in the isolated system. This value is slowly approached as the interchain distance increases, while, once the cutoff is applied to the Coulomb potential, the limit is reached as soon as the densities of the system and its periodic images do not interact. If we consider the convergence of the quasiparticle gap and of the binding energy with respect to the cell volume we notice that, if a cutoff is not used, the position of the absorption peak is controlled by the convergence of the Bethe-Salpeter equation 
(a)

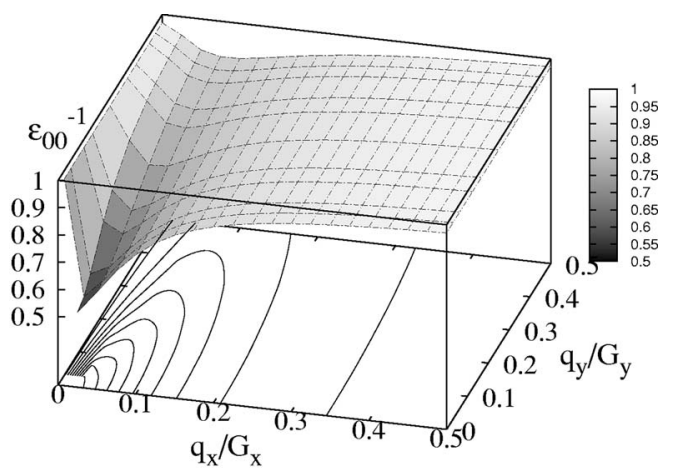

(b)

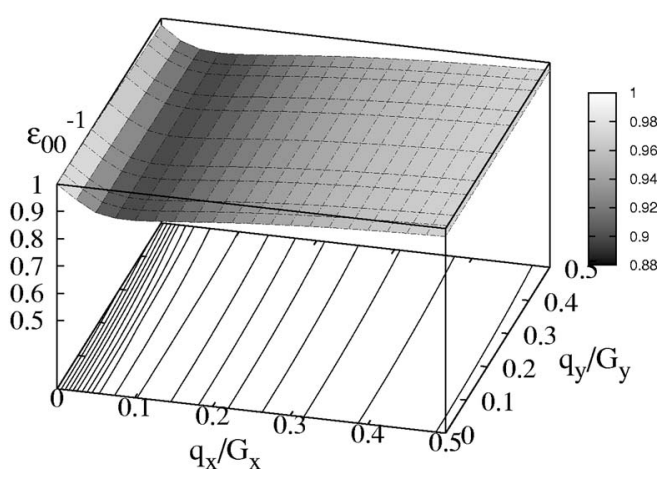

FIG. 8. Values of $\epsilon_{00}^{-1}\left(q_{x}, q_{y}\right)$ in the $q_{z}=0$ plane for the $H_{2}$ chain with an interchain distance of 25 a.u., using the bare Coulomb (top) and the cutoff potential (bottom). The axis of the chain is along the $x$ direction.

solution, which, in turn, depends on the (slower) convergence of the GW energies. It is clear from Fig. 6 that the use of the cutoff allows us to considerably speed up this bottleneck.

The use of our cutoff also has an important effect on the Brillouin zone sampling. In Fig. 8 we show the value of $\epsilon_{00}^{-1}\left(q_{x}, q_{y}\right)$ in the $q_{z}=0$ plane for a supercell corresponding to an interchain distance of 25 a.u. (13.23 $\AA$ ). When the cutoff potential is used (bottom panel) the screening is smaller, compared to the case of the bare potential (top panel). Looking at the direction perpendicular to the chain (the chain axis is along the $x$ direction) we see that the dielectric matrix is approximately constant, and this fact allows us to sample the Brillouin zone only in the direction of the chain axis. For both the GW and Bethe-Salpeter calculations a three dimensional sampling of the Brillouin zone is needed to get converged results when no cutoff is used, while a simple onedimensional sampling can be adopted when the interaction is cut off.

\section{CONCLUSIONS}

An infinite system is an artifact that allows us to exploit the powerful symmetry properties of an ideal system to approximate the properties of a finite one that is too large to be simulated at once. In order to use the valuable supercell approximation for systems that are periodic in less than three dimensions some cutoff techniques are required. The technique presented here provides a recipe to build the supercell, together with a Coulomb potential in Fourier space in such a way that the interactions with all the undesired images of the system are canceled. This technique is exact for the supercell sizes given and can in most cases be well approximated using supercells whose length is twice that of the system size in the nonperiodic dimensions. The method is very easily implemented in all available codes that use the supercell scheme, and is independent of the adopted basis set. We have tested it both in a real space code for LDA band-structure calculation of an atomic chain, and a plane wave code, for the static polarizability in RPA approximation, GW quasiparticle correction, and photoabsorption spectra in the BetheSalpeter scheme, showing that the convergence with respect to volume of the empty space needed to isolate the system from its images is greatly enhanced, and the sampling of the Brillouin zone is greatly reduced, being only necessary along the periodic directions of the system.

\section{ACKNOWLEDGMENTS}

This research was supported by EU Research and Training Network "Exciting" (Contract HPRN-CT-2002-00317), EC 6th framework Network of Excellence NANOQUANTA (NMP4-CT-2004-500198) and Spanish MEC. A.R. acknowledges the Foundation under the Bessel research award (2005). We thank Alberto Castro, Miguel A. L. Marques, Heiko Appel, Patrick Rinke, and Christoph Freysoldt for helpful discussions and friendly collaboration, and Eric Chang for having carefully proofread the manuscript.
${ }^{1}$ M. L. Cohen, Solid State Commun. 92, 45 (1994); Phys. Scr. 1, 5 (1982); J. Ihm, A. Zunger, and M. L. Cohen, J. Phys. C 12, 4409 (1979); W. E. Pickett, Comput. Phys. Rep. 9, 115 (1989); M. C. Payne, M. P. Teter, D. C. Allan, T. A. Arias, and D. Joannopoulos, Rev. Mod. Phys. 64, 1045 (1992).

${ }^{2}$ M. Frigo and S. G. Johnson, Proc.-IEEE Ultrason. Symp. 3, 1381 (1998)

${ }^{3}$ J. E. Lebowitz and E. H. Lieb, Phys. Rev. Lett. 22, 631 (1969).

${ }^{4}$ See, for example, A. P. Alivisatos, Science 271, 933 (1996); Carbon Nanotubes: Synthesis, Structure, Properties, and Applica- tions, edited by M. S. Dresselhaus, G. Dresselhaus, and Ph. Avouris (Springer Verlag, Berlin, New York, 2001); Encyclopedia of Nanoscience and Nanotechnology (American Scientific Publishers, Stevenson Ranch, CA, 2004); Handbook of Nanostructured Biomaterials and Their Applications in Nanobiotechnology, edited by H. S. Nalwa (American Scientific Publishers, Stevenson Ranch, CA, 2005); G. Burkhard, H.-A. Engel, and D. Loss, Fortschr. Phys. 48, 965 (2000).

${ }^{5}$ The maximum value of the size of the cutoff axial length $h$ both in Ref. 35 and in Eq. (29) is $2 \pi / \Delta k$, where $\Delta k$ is the spacing of 
the $k$ point grid in the direction of the cylinder axis.

${ }^{6}$ L. Hedin, Phys. Rev. 139, 796 (1965); L. Hedin and S. Lundqvist, Solid State Phys. 23, 1 (1969); F. Aryasetiawan and O. Gunnarson, Rep. Prog. Phys. 61, 237 (1998); W. G. Aulbur, L. Jönsson, and J. W. Wilkins, Solid State Phys. 54, 1 (1999).

${ }^{7}$ G. Onida, L. Reining, and A. Rubio, Rev. Mod. Phys. 74, 601 (2002), and references therein.

${ }^{8}$ Sottile, F. Bruneval, A. G. Marinopoulos, L. Dash, S. Botti, V. Olevano, N. Vast, A. Rubio, and L. Reining, Int. J. Quantum Chem. 112, 684 (2005).

${ }^{9}$ M. Leslie and M. J. Gillian, J. Phys. C 18, 973 (1985).

${ }^{10}$ B. Wood, W. M. C. Foulkes, M. D. Towler, and N. D. Drummond, J. Phys.: Condens. Matter 16, 891 (2004).

${ }^{11}$ G. Shaviv and N. J. Shaviv, J. Phys. A 36, 6187 (2003).

${ }^{12}$ D. P. Tieleman, S. J. Marrink, and H. J. C. Berendsen, Biochim. Biophys. Acta 1331, 235 (1997); D. P. Tieleman, ibid. 5, 10 (2004).

${ }^{13}$ Y. Fujiwara, T. Fujita, M. Kohno, C. Nakamoto, and Y. Suzuki, Phys. Rev. C 65, 014002 (2002).

${ }^{14}$ G. Makov and M. C. Payne, Phys. Rev. B 51, 4014 (1995).

${ }^{15}$ S. W. Deleeuw, J. W. Perram, and E. R. Smith, Proc. R. Soc. London, Ser. A 373, 27 (1980).

${ }^{16}$ P. P. Ewald, Ann. Phys. 64, 253 (1921).

${ }^{17}$ E. Spohr, J. Chem. Phys. 107, 6342 (1994).

${ }^{18}$ I. Yeh and M. Berkowitz, J. Chem. Phys. 111, 3155 (1999).

${ }^{19}$ G. J. Martyna and M. E. Tuckerman, J. Chem. Phys. 110, 2810 (1999).

${ }^{20}$ A. Bródka, Mol. Phys. 61, 3177 (2003).

${ }^{21}$ D. M. Heyes, M. Barber, and J. H. R. Clarke, J. Chem. Soc., Faraday Trans. 2 73, 1485 (1977).

${ }^{22}$ A. Grzybowski, E. Gwóźdź, and A. Bródka, Phys. Rev. B 61, 6706 (2000).

${ }^{23}$ P. Mináry, M. E. Tuckerman, K. A. Pihakari, and G. J. Martyna, J. Chem. Phys. 116, 5351 (2002).

${ }^{24}$ P. Mináry, J. A. Morrone, D. A. Yarne, M. E. Tuckerman, and G. J. Martyna, J. Chem. Phys. 121, 11949 (2004).

${ }^{25}$ L. Greengard, The Rapid Evaluation of Potential Fields in Particle Systems (MIT, Cambridge, MA, 1987).

${ }^{26}$ A. Castro, A. Rubio, and M. J. Stott, Can. J. Phys. 81, 1 (2003).

${ }^{27}$ K. N. Kudin and G. E. Scuseria, J. Chem. Phys. 121, 2886
(2004).

${ }^{28}$ P. A. Schultz, Phys. Rev. B 60, 1551 (1999).

${ }^{29}$ R. W. Hockney and J. W. Eastwood, Computer Simulations Using Particles (McGraw-Hill, New York, 1981).

${ }^{30}$ M. R. Jarvis, I. D. White, R. W. Godby, and M. C. Payne, Phys. Rev. B 56, 14972 (1997).

${ }^{31}$ M. A. L. Marques, A. Castro, G. F. Bertsch, and A. Rubio, Comput. Phys. Commun. 151, 60 (2003).

${ }^{32}$ G. Onida, L. Reining, R. W. Godby, R. Del Sole, and W. Andreoni, Phys. Rev. Lett. 75, 818 (1995).

${ }^{33}$ I. S. Gradshteyn and M. Rhysik, Tables of Integrals, Series and Products (Academic, New York, 1980).

${ }^{34}$ J. D. Jackson, Classical Electrodynamics (Wiley, New York, 1999).

${ }^{35}$ C. Spataru, S. Ismail-Beigi, L. X. Benedict, and S. G. Louie, Appl. Phys. A 78, 1129 (2004).

${ }^{36}$ http://people.roma2.infn.it/ marini/self

${ }^{37}$ N. Troullier and J. L. Martins, Phys. Rev. B 43, 1993 (1991).

${ }^{38}$ J. P. Perdew and A. Zunger, Phys. Rev. B 23, 5048 (1981).

${ }^{39}$ S. Albrecht, L. Reining, R. Del Sole, and G. Onida, Phys. Rev. Lett. 80, 4510 (1998); L. X. Benedict, E. L. Shirley, and R. B. Bohn, ibid. 80, 4514 (1998); M. Rohlfing and S. G. Louie, ibid. 81, 2312 (1998); Phys. Rev. B 62, 4927 (2000).

${ }^{40}$ S. L. Adler, Phys. Rev. 126, 413 (1962); N. Wiser, Phys. Rev. 129, 62 (1963).

${ }^{41}$ C. D. Spataru, S. Ismail-Beigi, L. X. Benedict, and S. G. Louie, Phys. Rev. Lett. 92, 077402 (2004).

${ }^{42} \mathrm{http}: / /$ www.abinit.org

${ }^{43}$ R. W. Godby and R. J. Needs, Phys. Rev. Lett. 62, 1169 (1989). To get converged inverse dielectric matrix we have used unoccupied bands up to $28 \mathrm{eV}$ in the electron-hole energies, and a number of $G$ vectors until $G^{2} / 2=50 \mathrm{eV}$ in the inversion of the matrix.

${ }^{44}$ M. S. Hybertsen and S. G. Louie, Phys. Rev. Lett. 55, 1418 (1985); R. W. Godby, M. Schluter, and L. J. Sham, Phys. Rev. B 37, 10159 (1988).

${ }^{45}$ C. Delerue, G. Allan, and M. Lannoo, Phys. Rev. Lett. 90, 076803 (2003).

${ }^{46}$ D. Varsano, A. Marini, and A. Rubio (unpublished). 